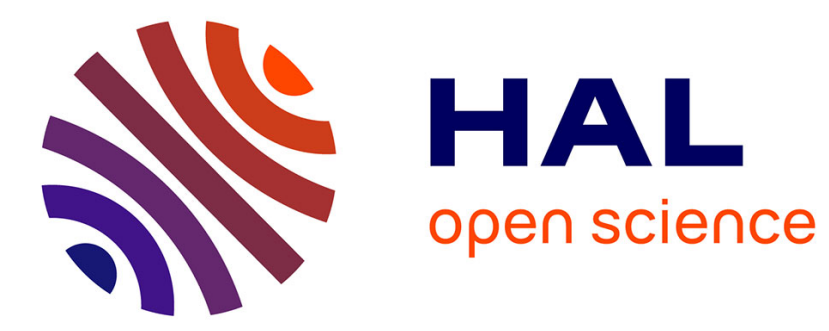

\title{
STRUCTURE DE BANDES ÉLECTRONIQUE DE GaSe
}

\author{
A. Bourdon
}

\section{To cite this version:}

A. Bourdon. STRUCTURE DE BANDES ÉLECTRONIQUE DE GaSe. Journal de Physique Colloques, 1974, 35 (C3), pp.C3-261-C3-267. 10.1051/jphyscol:1974338 . jpa-00215586

\section{HAL Id: jpa-00215586 https://hal.science/jpa-00215586}

Submitted on 1 Jan 1974

HAL is a multi-disciplinary open access archive for the deposit and dissemination of scientific research documents, whether they are published or not. The documents may come from teaching and research institutions in France or abroad, or from public or private research centers.
L'archive ouverte pluridisciplinaire HAL, est destinée au dépôt et à la diffusion de documents scientifiques de niveau recherche, publiés ou non, émanant des établissements d'enseignement et de recherche français ou étrangers, des laboratoires publics ou privés. 


\title{
STRUCTURE DE BANDES ÉLECTRONIQUE DE GaSe
}

\author{
A. BOURDON \\ Université de Paris VI, Laboratoire de Luminescence II (*) \\ 4, place Jussieu, 75230 Paris Cedex 05, France
}

\begin{abstract}
Résumé. - GaSe est un semiconducteur dont la structure lamellaire s'explique par la noncomplémentarité des valences (3 et 6 ) des composants. Un modèle à uné couche rend déjà compte de nombreuses propriétés, en particulier la $\mathrm{BV}$ et la $\mathrm{BC}$ sont dues à des états liants ou antiliants assez localisés autour du pont $\mathrm{Ga}-\mathrm{Ga}$ interne aux couches, ce qui explique les règles de sélection observées pour les transitions directes et indirectes. Pour. un calcul précis de structure de bandes pour un cristal à 3 dimensions, nous avons utilisé une méthode de pseudo-potentiel dérivée de celle de Lin et Kleinman. La transition fondamentale directe a lieu en $\Gamma$ tandis que l'indirecte se fait entre des maxima de la bande de valence situés au voisinage de $K$ et $\Gamma$ pour la bande de conduction. Cette position de la transition indirecte rend bien compte de l'invariance de Egd-Egi par rapport à la température ou à la structure cristalline. La légère bosse dans le spectre d'absorption au voisinage de la transition fondamentale est expliquée par une transition indirecte résonnante. Nous trouvons des masses réduites peu anisotropes pour l'exciton $m_{x y}=0,12 m_{0}$ et $m_{z}=0,22 m_{0}$ au lieu des valeurs expérimentales 0,15 et $0,36 m_{0}$. L'accord est bon entre $2 \mathrm{et} 9 \mathrm{eV}$ entre la structure de bandes proposée et les spectres de réflectivité.
\end{abstract}

Abstract. - The non-complementarity of valencies of $\mathrm{GaSe}$ (3 and 6) explains its layer structure. $A$ one-layer model makes some experimental properties clear. For instance, the VB and CB come from bonding and antibonding states which are located (inside the layer) near the coupled atoms of gallium ; this explains the selection rules observed for direct and indirect transitions. We used a pseudo-potential method derived from Lin and Kleinman's to compute the electronic band structure of GaSe. The initial and final states of the fundamental direct transition are both in $\Gamma$ while for the indirect one, the maxima of the VB are located near the $K$ points and the minimum of the $\mathrm{CB}$ is in $\Gamma$. This model of indirect transition accounts for the invariance of Egd-Egi versus temperature and crystal structure. The slight hump above the absorption edge is explained by an indirect resonant transition. Our reduced-mass values for the exciton are rather little anisotropic and in good accordance with experimental values : $m_{x}=m_{y}=0.12 m_{0}$ and $m_{z}=0.22 m_{0}$ instead of 0.15 and $0.36 m_{0}$. Our band structure model explains well previously published reflectivity spectra.

1. Introduction. - Parmi les semiconducteurs connus, les composés binaires III-VI ont été le plus tardivement étudiés. Cette réticence devant ces corps provient-elle d'une sorte de répulsion théorique devant un composé qui ne respecterait pas l'habituelle complémentarité des valences des composants, ou bien, plus prosajquement, les chercheurs n'ont-ils pas eu peur de faire des expériences sur des corps très friables ? En effet, ces corps cristallisent sous forme lamellaire et l'on doit s'attendre à ce que leurs propriétés physiques présentent une certaine anisotropie. GaS et surtout GaSe ont été les plus étudiés.

GaSe cristallise en couches à l'intérieur desquelles les atomes sont rattachés par des fortes liaisons (covalentes en majorité); par contre, les liaisons entre couches sont de type Van der Waals. C'est pourquoi les premiers calculs de structure de bande de Bassani et al. [1] d'une part et de Kamimura [2] d'autre part ont porté sur une couche isolée (cristal à deux dimensions).

(*) Equipe de Recherche associée au C.N.R.S.
Cette simplification peut être fructueuse, mais ces auteurs ont fait une supposition contestable sur la nature de la bande de conduction qui est d'ailleurs infirmée par l'expérience [3-4]. Les résultats présentés ici ont fait l'objet d'une thèse soutenue en 1971 [5]. Ils se rapprochent un peu de ceux de Schlüter [6-7] qui a aussi fait ses calculs par une méthode de pseudopotentiel sur un cristal à 3 dimensions.

\section{Composés III-VI, liaison covalente et nature du} gap. - Si l'on examine une couche isolée de GaSe, on peut considérer qu'elle provient de l'accolement de deux réseaux plans hexagonaux, analogues à ceux du graphite, où, dans chacun, tout atome de Se est entouré de trois atomes de $\mathrm{Ga}$ et inversement; ces deux sous-couches sont reliées entre elles par des. ponts Ga-Ga qui déforment les deux réseaux hexagonaux (Fig. 1).

On peut fort simplement expliquer cette structure de couche épaisse par des raisonnements de chimie très élémentaire. Dans une demi-couche, qui est un réseau à deux dimensions, la maille élémentaire de ce. 

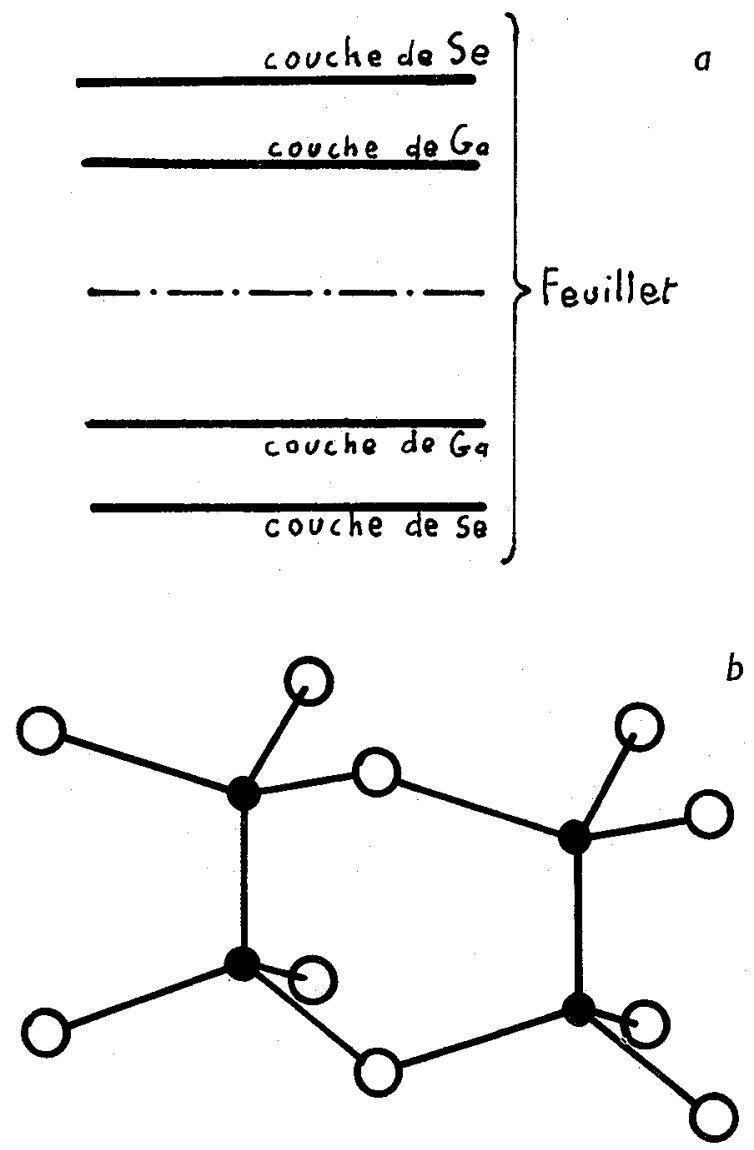

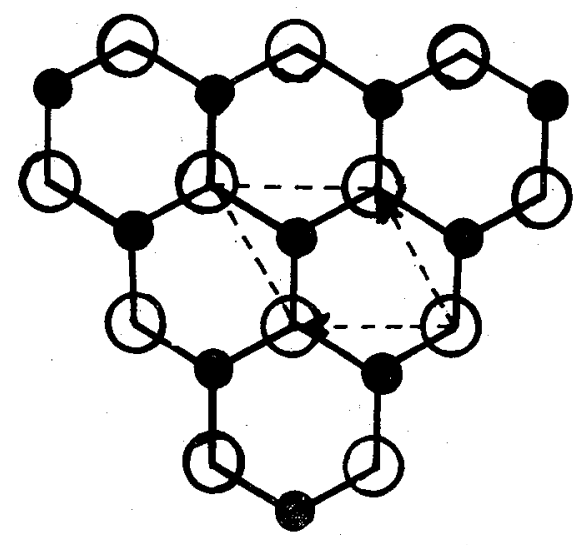

c

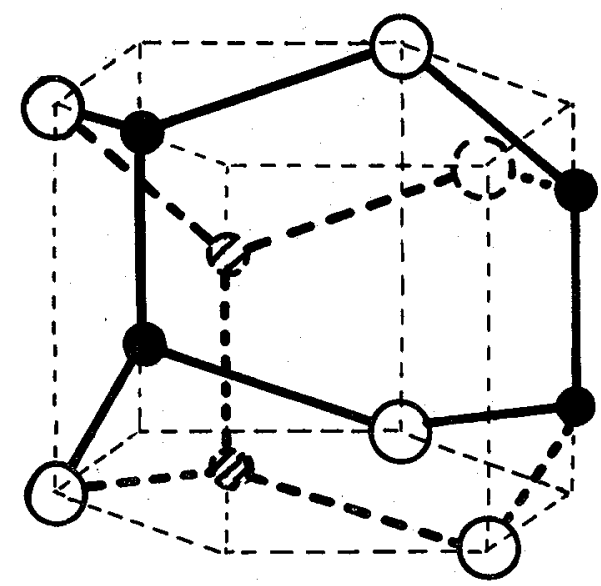

Frg. 1. - Structure du feuillet. $\bigcirc$ atome de sélénium; atome de gallium, a) Représentation en couches; b) Vue en perspective d'une portion de feuillet; c) Vue en plan du réseau hexagonal; $d$ ) Déformation « en chaise " des réseaux hexagonaux.

réseau contient une molécule de GaSe, c'est-à-dire 9 électrons externes dont 6 servent à relier les atomes entre eux si l'on admet que les liaisons sont covalentes. Que deviennent les 3 électrons restants ? Comme Se est plus électronégatif que $\mathrm{Ga}$, il complète son octet en prenant deux électrons, alors que Ga n'en reçoit qu'un. Tous les atomes de gallium d'une demi-couche ont donc une valence libre et peuvent de ce fait être reliés aux atomes de gallium d'un réseau hexagonal voisin. On pourrait imaginer que ces liaisons transversales Ga-Ga ne soient pas toutes dirigées du même côté d'une « demi »-couche, l'énergie de cette structure ne serait sans doute pas minimum.

Toujours dans l'hypothèse de pures liaisons covalentes à l'intérieur des couches, il ne resterait plus que la possibilité de liaisons de Van der Waals entre différentes couches. En réalité, il semble que GaSe soit très légèrement ionique, GaS l'étant un peu plus.

Ainsi la structure en couches épaisses et la grande facilité de clivage sont-elles facilement expliquées par la présence de 9 électrons périphériques dans une molécule de GaSe. Nous allons voir que la nature de la transition fondamentale s'en déduit aisément.

Les électrons de la liaison $\mathrm{Ga}-\mathrm{Ga}$ sont les moins solidement ancrés et forment la plus haute bande de valence. Comme cette liaison est évidemment la plus fragile, les états de la bande de conduction sont formés d'états antiliants Ga-Ga. Au point $\Gamma$ de la zone de Brillouin, les bandes de valence et de conduction correspondent à des états respectivement pair et impair par rapport au plan de symétrie de la couche. L'expérience confirme bien la différence de parité de la BC et de la BV. En effet l'absorption est beaucoup plus intense (d'environ un facteur 16) en polarisation perpendiculaire aux couches qu'en polarisation parallèle à celles-ci [3-4]. Pour s'en convaincre, il suffit de regarder à l'œil nu la lumière jaune d'une lampe à sodium traversant une lamelle de GaSe d'environ $40 \mu$ d'épaisseur. Lorsque celle-ci est inclinée par rapport à la direction de propagation, on observe facilement cette différence d'absorption au moyen d'un polaroïd. La règle de sélection n'est pas totalement respectée, car les effets de spin et, dans une moindre mesure, les effets de certains types d'empilement de couches (structure $\gamma$ ) mélangent un peu des états de parité opposée. Dans les modèles proposés antérieurement, la bande de conduction et la bande de valence étaient toutes deux paires par rapport à la symétrie plane 
pour rendre compte, par une transition dipolaire, de l'absorption optique dans les conditions expérimentales les plus faciles à réaliser, c'est-à-dire lorsque la lumière arrive perpendiculairement aux couches.

La nature des bandes électroniques au voisinage de la bande interdite est donc explicable dans un modèle à deux dimensions seulement.

3. Calculs du modèle à trois dimensions. - Nous avons fait appel, pour le calcul des énergies des bandes électroniques, à une méthode de pseudo-potentiel qui nécessite un développement en ondes planes des fonctions propres de l'hamiltonien. Si dans nos calculs nous avons négligé les effets de couplage LS, nous avons en réalité utilisé une base composée de fonctions propres des différentes représentations du groupe d'espace du cristal.

Toutes les structures cristallines de GaSe sont construites par empilement de couches élémentaires bidimensionnelles identiques. Deux couches adjacentes peuvent s'obtenir l'une de l'autre, soit par translation oblique, soit par inversion. On constate que dans un monocristal les empilements sont toujours du même type. La structure cristalline où les empilements se font par inversion est unique (structure $\beta$ ) et la maille élémentaire contient deux couches. Il existe par contre plusieurs structures cristallines à empilements par translation : structure $\varepsilon$ à deux couches, structure $\gamma$ à trois couches, etc... structures que nous qualifierons, d'une manière générale, de parallèle. Il semble évident que, en gros, toutes ces structures cristallines ont les mềmes structures de bandes électroniques dans un schéma de zone étendue, mais que, par contre, dans la zone de Brillouin, elles dépendent de la structure cristalline. Pour avoir une meilleure convergence dans nos calculs, il a fallu prendre les structures cristallines à maille élémentaire la moins volumineuse possible :
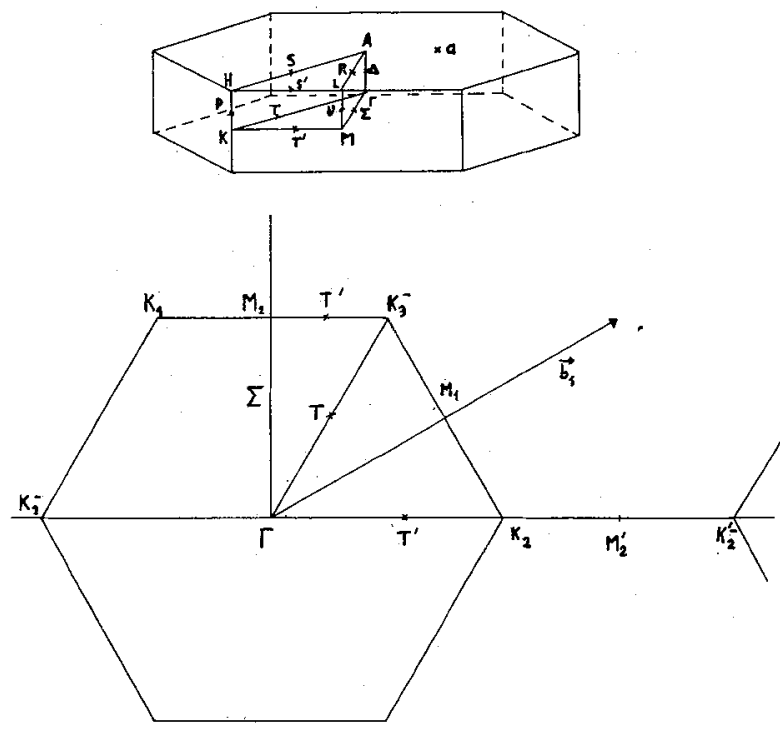

FIG. 2. - Zone de Brillouin de la structure $\beta$. $a$ : Point courant des faces horizontales de la zone de Brillouin. ce sont les structures $\beta$ et $\varepsilon$ à deux couches. Puisque les couches élémentaires sont faiblement couplées, les bandes électroniques des structures $\beta$ et $\varepsilon$ se présenteront par paires dans la zone de Brillouin. L'écart d'énergie entre chacune des deux bandes de la paire est en quelque sorte une mesure du "produit de la probabilité de présence des électrons entre les couches par le potentiel d'interaction entre celles-ci is. Comme les atomes de sélénium sont sur les faces externes des couches, on a une indication sur la répartition des électrons entre le gallium et le sélénium. Nos calculs ont surtout été faits sur la structure $\beta$ (groupe $\mathrm{D}_{6 \mathrm{~h}}^{4}$ ). qui présente une plus grande symétrie que la structure $\varepsilon$ $\left(\mathrm{D}_{3 \mathrm{~h}}^{1}\right)$.

Nous avons employé un pseudo-potentiel dérivé de celui proposé par Lin et Kleinman [8]. Le potentiel proposé par ces auteurs est analytiquement très simple. Puisque lẹs états de cœur forment une base presque complète dans les régions profondes de l'atome, ils ont supposé que la compensation y était totale, alors qu'à l'extérieur celle-ci n'était que partielle.

Pour la partie locale :

$$
W_{\mathrm{e}}=0 \quad \text { pour } r<r_{0}
$$

et

$$
W_{\mathrm{e}}=\frac{Z}{r}\left(1-\mathrm{e}^{-B\left(r-r_{0}\right)}\right) \text { pour } r>r_{0}
$$

$Z$ étant une charge effective ressentie par les électrons externes.

La partie non locale de ce pseudo-potentiel est un opérateur qui élève l'énergie des composantes $s$ des fonctions sur lesquelles il agit, car dans le cœur, les fonctions $s$ ont une énergie inférieure à celle des fonctions $p$. Nous avons repris sans la changer la partie non locale de ce pseudo-potentiel tandis que, pour la partie locale, la variable $Z$ était remplacée par les valeurs des valences du gallium et du sélénium. L'autocohérence était alors assurée par l'emploi d'une constante diélectrique $\varepsilon(k, 0)$ anisotrope dérivée de celle proposée par Penn [9] dans laquelle figurent les constantes diélectriques statiques fournies par l'expérience

$$
\begin{gathered}
\varepsilon_{0} / /=7,8 \quad \text { et } \quad \varepsilon_{0} \perp=10 \\
\varepsilon(K)=1+\left[\left(\varepsilon_{0} / /-1\right)^{2} \sin ^{2} \theta+\right. \\
\left.+\left(\varepsilon_{0} \perp-1\right)^{2} \cos ^{2} \theta\right]^{1 / 2}\left(1+A K^{2}\right)^{-2}
\end{gathered}
$$

$A$ étant un paramètre ajustable $(A=0,487)$ et $\theta$ étant l'angle que forme $K$ avec le plan des couches. Pour la partie non locale [8], les valeurs de $\alpha$. sont de 0,210 pour le gallium et 0,487 pour le sélénium.

4. Structure de bandes proposée. - Avec les valeurs des 3 paramètres ci-dessus, nous obtenons un échelónnement des bandes qui correspond bien à notre modèle covalent (Fig. 3 et 4).

- Les 4 bandes les plus profondes sont localisées sur Se $(s)$; 

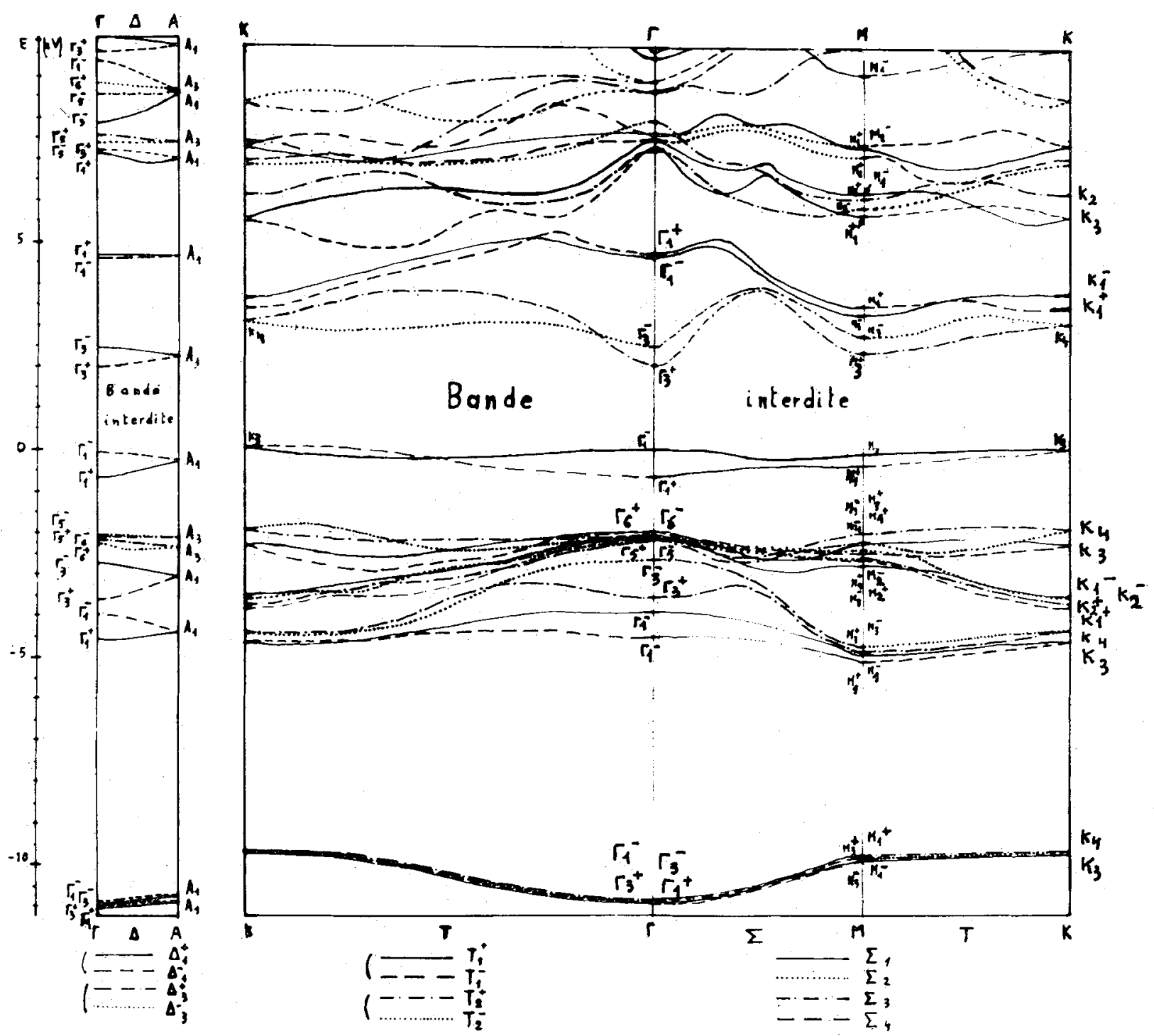

Fig. 3. - Structure de bandes en $K_{z}=0$.

- 4 bandes $p_{z}(\mathrm{Se})$ et $s(\mathrm{Ga})$ assez délocalisées ;

- 8 bandes $p_{x}$ et $p_{y}$ très localisées dans le feuillet;

- 4 bandes $p_{z}-s$ dont les deux premières $\left(\Gamma_{1}^{+}\right.$ et $\Gamma_{1}^{-}$) sont les plus hautes bandes de valence et sont bien séparées des deux autres $\left(\Gamma_{3}^{+}\right.$et $\left.\Gamma_{3}^{-}\right)$qui forment les premières bandes de conduction. Les fonctions $\Gamma_{1}$ et $\Gamma_{3}$ sont respectivement paires et impaires par rapport au plan de symétrie. Ces fonctions sont relativement bien localisées sur le gallium.

- Ensuite les autres bandes de conduction.

Cette structure de bandes permet de bien expliquer les résultats expérimentaux d'origine optique concernant GaSe. Elle présente en outre des aspects nouveaux concernant les porteurs de charge qui ne sont ni vérifiés ni infirmés. La transition directe fondamentale a lieu en $\Gamma$ et a bien l'énergie de $2,17 \mathrm{eV}$ et obéit bien à la règle de sélection présentée plus haut. Les effets de spin ont un double rôle: ils élèvent l'énergie des fonctions $\Gamma_{5}^{ \pm}$, et $\Gamma_{6}^{ \pm}$et la rapprochent encore plus de $\Gamma_{1}^{-}$; ils permettent de plus à la fonction $\Gamma_{1}^{-}$de se combiner partiellement avec la fonction $\Gamma_{6}^{-}$ce qui permet la légère absorption directe en lumière polarisée parallèlement aux couches.

Dans ce modèle, les maxima absolus de la bande de valence sont situés, si les coordonnées de $K$ sont $\left(\frac{1}{3}, \frac{1}{3}, 0\right)$, aux points suivants :

- le long de $T$ entre $\Gamma$ et

$$
K\left(\frac{0,80}{3}, \frac{0,80}{3}, 0\right)
$$

— et le long de $T^{\prime}$ entre $K$ et

$$
M\left(\frac{1-0,36}{3}, \frac{1+0,18}{3}, 0\right)
$$

tandis que le minimum absolu de la bande de conduction se trouve en $\Gamma$. Le gap indirect est bien de $2,117 \mathrm{eV}$. Les modèles présentés antérieurement $(1,2,7)$ 

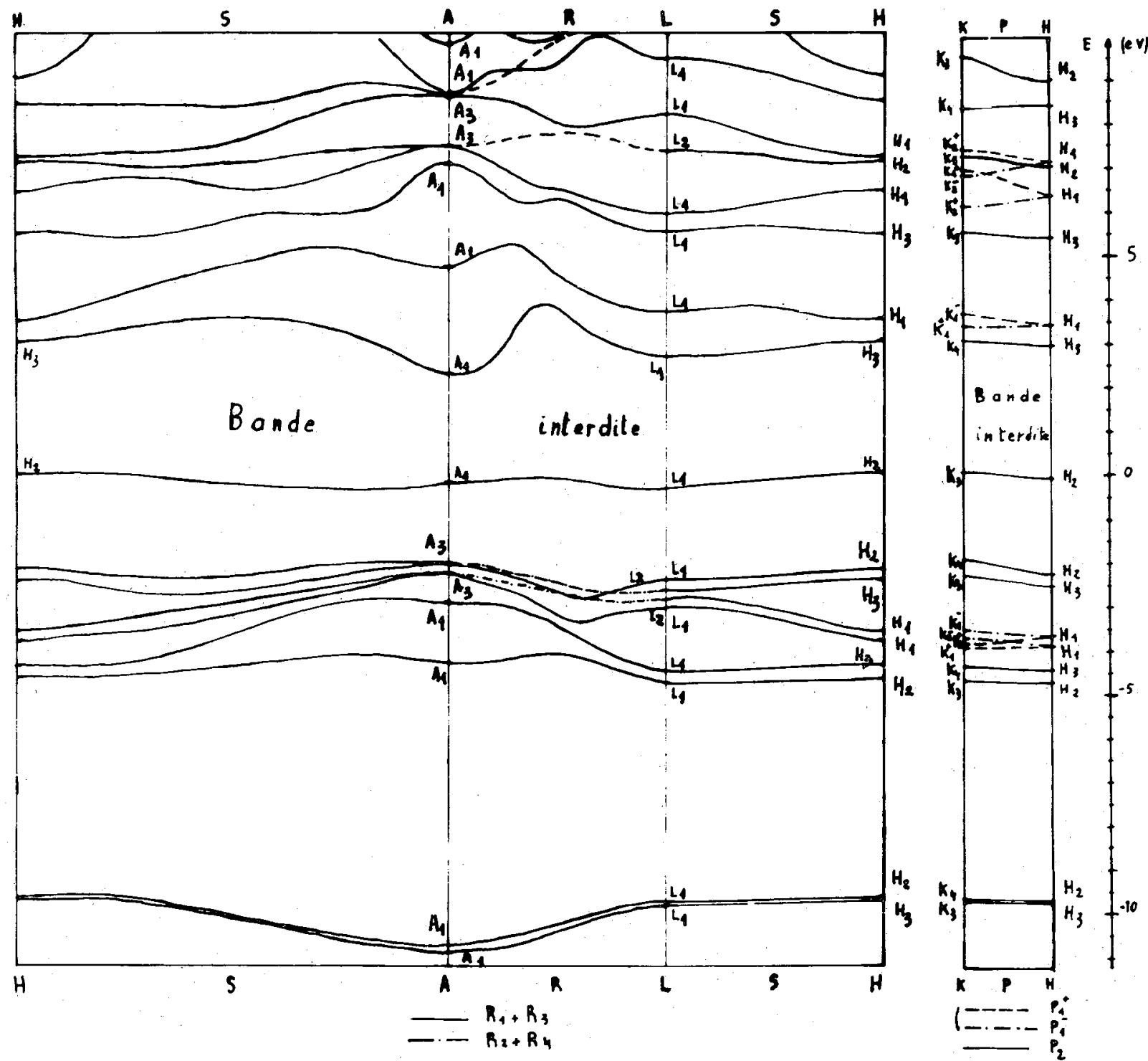

Fig. 4. - Structure de bandes en $K_{z}=\pi / c$.

plaçaient tous le maximum de la bande de valence en $\Gamma$ et le minimum de la bande de conduction en $M$. L'écart en énergie de $53 \mathrm{meV}$ entre les valeurs du gap direct et indirect provient de la bande de valence dans notre modèle tandis que, dan's les autres, il trouve son origine dans la bande de conduction. Or, il se trouve que cet écart d'énergie est indépendant de la température [11] et de la structure cristalline [12]. Il semble plus naturel de supposer que c'est la bande de valence qui est la moins sensible à ces variations, car elle est plus localisée dans les couches. La règle de sélection en transition indirecte est bien la même qu'en transition directe car les transitions électromagnétiques dipolaires virtuelles y obéissent aussi bien autour de $\Gamma$ que de $K$.

A $10 \mathrm{meV}$ au-dessus du bord de bande, il a été observé une bosse dans le spectre d'absorption que l'on a interprétée comme étant due à des excitons bidimensionnels. Comme nous le verrons pour les masses effectives de porteurs, ce caractère bidimensionnel est à exclure. Si l'on n'envisage pas la présence permanente d'impuretés, on ne peut trouver non plus d'anomalie dans la densité conjointe d'états. Il reste la possibilité d'une anomalie dans l'élément de matrice de transition. Peut-être est-elle due à des transitions indirectes résonnantes (transitions au cours desquelles il y a pratiquement conservation de l'énergie pour les deux transitions virtuelles). L'écart d'énergie entre la bosse et le premier exciton est de $240 \mathrm{~cm}^{-1}$, valeur comparable au $253,8 \mathrm{~cm}^{-1}$ trouvé en effet Raman [14].

Les masses effectives calculées sont très peu anisotropes et parfois même l'anisotropie est inverse de celle à laquelle on pourrait s'attendre. La masse effective des trous dans les 12 «poches» entourant les points $K$ a été calculée le long des axes $T$ et $T^{\prime}$, mais il n'a pas été possible de calculer l'autre composante horizontale dans la direction perpendiculaire à ces axes, elle doit être du même ordre de grandeur (Tableau I). 


\section{TABLEAU I}

\section{Masses effectives des trous} et des électrons

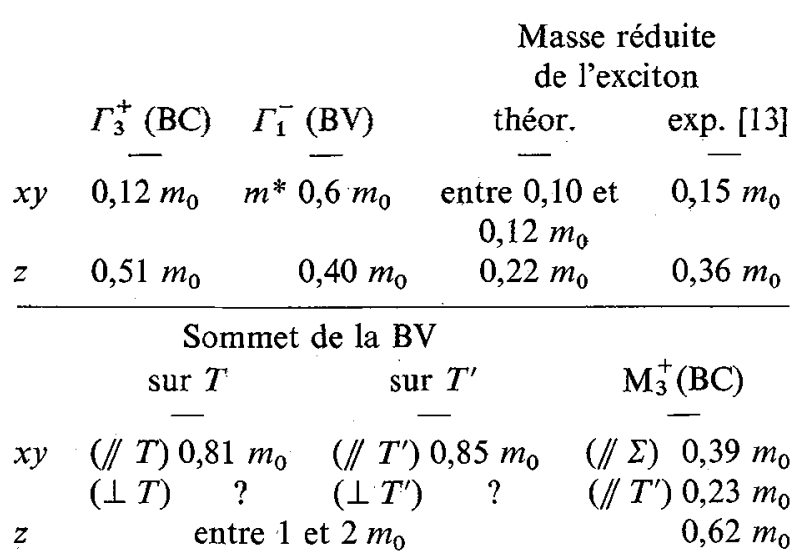

La grande densité d'états de porteur de type $p$ due aux douze poches de trous, contre une poche d'électron, compense sans doute leur faible mobilité, ce qui permet d'expliquer l'observation à haute température d'une conduction de type $p$. Pour comparer ce modèle avec les spectres de réflectivité de Bassani et al. [10], il aurait fallu calculer la densité conjuguée d'états et en tirer les maxima ou les singularités (Fig. 5). A partir des figures 3 et 4 on peut malgré tout les deviner.

4.1 TRANSITION OU $E$ EST PERPENDICULAIRE A L'AXE TERNAIRE. - C'est dans ces conditions d'expérience que les mesures les plus fines ont été faites. Dans notre modèle nous trouvons donc les extréma de densités d'états à :

- 3,09 et 3,11 eV : Les transitions correspondantes $\mathrm{M}_{1}^{+} \rightarrow \mathrm{M}_{3}^{-}$et $\Gamma_{1}^{+} \rightarrow \Gamma_{3}^{-}$doivent être visibles car la

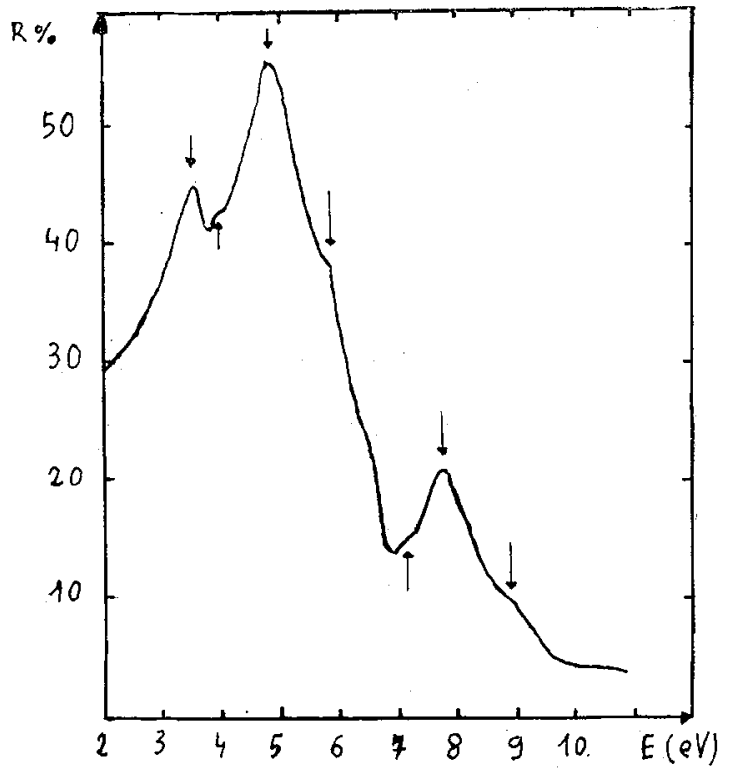

FIg. 5. - Courbe de réflectivité du GaSe. densité d'états doit être forte (points hyperboliques). Ces transitions sont permises et relativement intenses à cause du fort couplage entre les bandes de valence $s-p_{z}$ et $p_{x}-p_{y}$.

- De 3,32 à 3,62 eV : Deux paires de bandes $\mathrm{T}_{1}^{ \pm}$ restent parallèles l'une à l'autre entre $M_{1}^{ \pm}$et $K_{3}$ ou $K_{1}^{ \pm}$.

- A 4,1 eV : Transition $\Gamma_{6}^{-} \rightarrow \Gamma_{3}^{+}$.

- A $4,7 \mathrm{eV}$ : Transition $\Gamma_{6}^{+} \rightarrow \Gamma_{3}^{-}$(point hyperbolique).

- De 4,6 eV à 6,2 eV : La densité d'états conjuguée entre $M$ et $K$ présente de nouveau de très grandes valeurs :

- Un grand nombre de bandes de valence $T$ de type $p_{x}-p_{y}$ sont parallèles aux quatre premières. bandes de conduction. On doit trouver le maximum vers $5 \mathrm{eV}$ à la transition $\mathrm{K}_{4} \rightarrow \mathrm{K}_{4}$.

- Le long de cette même droite et dans la même gamme d'énergie, on trouve aussi un grand nombre de transitions possibles entre les 2 dernières bandes de valence et la branche inférieure des bandes de conduction de type $p_{x}$ et $p_{y}\left(\mathrm{~K}_{3} \rightarrow \mathrm{K}_{3}\right)$.

- Entre 7,6 et $8 \mathrm{eV}$ : On trouve des transitions principalement entre les dernières bandes de valence et la plus haute branche des bandes de conduction de type $p_{x}-p_{y}$ et ceci aussi bien autour de $\Gamma$ qu'entre $M$ et $K$.

4.2 Polarisation parallèle a $c$. - Les paires de bandes de valence et de conduction sont bien de symétrie opposée par rapport au plan médian, mais la densité d'états conjuguée ne devrait pas présenter d'autre singularité que celle à $3,11 \mathrm{eV}$ en $\Gamma$ ou $M$, ce qui explique la variation régulière du taux de réflectivité au-dessus de $\mathrm{E}_{\mathrm{g}}$. Les transitions vers $3,6 \mathrm{eV}$ sont moins marquées qu'en $\mathrm{E} \perp c$, car elles sont dues à des effets de spin tandis qu'autour de $5 \mathrm{eV}$ on retrouve le même paquet de transitions qui sont permises pour toute direction de polarisation. Nous retrouvons le même pic à $5 \mathrm{eV}$ qu'en polarisation perpendiculaire à l'axe ternaire, mais décalé de $0,3 \mathrm{eV}$ vers les hautes énergies.

En résumé, nous retrouvons bien les spectres de réflectivité de Bassani. Presque toutes les transitions observées ont lieu dans les faces latérales de la zone de Brillouin entre états $s-p_{z}$ et $p_{x}-p_{y}$. Nous avons vu que les deux grosses bandes de réflectivité autour de 5 et $7,8 \mathrm{eV}$ sont dues à une somme de transitions qu'il sera impossible de distinguer expérimentalement. Il est presque surprenant que ces calculs arrivent même à expliquer le pic à $7,8 \mathrm{eV}$; peut-être sont-ils fiables pour les plus hautes bandes de conduction.

Remerciements. - Je remercie vivement $M$. le Professeur Mattler qui m'a permis de faire ce travail et je veux exprimer toute ma reconnaissance à $M$. le Professeur Hulin pour toute son aide scientifique et morale. 


\section{Bibliographie}

[1] Bassani, F. et Pastori-Parravicini, G., Nuovo Cimento LB (1967), $\mathrm{n}^{\circ} 1$.

[2] Kamimura, H., Nakao, K. et Nishina, Y., Phys. Rev. Lett. 22 (1969) 1379.

[3] Antoci, S. et Minich, L., Solid State Commun. 12 (1973) 649.

[4] Bourdon, A. et Khelladi, F., Solid State Commun. 9 (1971) 1715.

[5] Bourdon, A., Thèse de $3^{\mathrm{e}}$ cycle, Univer. Paris VI (1971).

[6] Schlueter, M., Colloque de Menton (1971).

[7] Schlueter, M., Rapport de la session de la Société Suisse de Physique 45 (1972) 73.
[8] Lin P. J. et Kleinman, L., Phys. Rev. 142 (1966) 478.

[9] Penn, D. R., Phys. Rev. 128 (1962) 2093.

[10] Bassani, F, Greenaway, D. L. et Fischer, G., Conf. sur les semiconducteurs (Dunod), Paris 1964, p. 51.

[11] Khelladi, F., Thèse de $3^{\mathrm{e}}$ cycle, Univer. Paris VI (1971).

[12] Aulich, E., Brebner, J. L. et Mooser, E., Phys. Stat. Sol. 31 (1969) 129.

[13] Halpern, J., Proc. Conf. Semicond., Tokio (1966), $J$ Phys. Soc. Japan 21, suppl. (1966) 180.

[14] Wright, G. B., Mooradian, A., Bull. Am. Phys. Soc. $11(1966) 812$.

\section{DISCUSSION}

C. Gout. - Le pseudo-potentiel utilisé est-il sphérique?

A. BouRdon. - Oui, et c'est la raison pour laquelle l'interaction entre couches a été peut-être surestimée.

Y. PETROFF. - Je crois que ce calcul a le très netavantage de tenir compte des liaisons entre couches (ce qui n'était pas le cas dans ceux de Bassani et al., Kao et Kamimura). Toutefois, je ne pense pas que l'on puisse attacher trop d'importance à cette valeur de $53 \mathrm{meV}$ entre le gap direct et indirect.

A. Bourdon. - Cette valeur de $53 \mathrm{meV}$, par sa constance dans les différentes structures [12] est au contraire très importante ; elle indique que les états correspondant à cette différence d'énergie sont très localisés dans la couche ce qui est le cas de la bande de valence et moins celui de la bande de conduction.
$\mathbf{M}^{\text {me }}$ RoBin. - Sur quel spectre de réflexion basezvous l'interprétation de la structure de bande proposée ? Etudié par qui ?

A. Bourdon. - Je me suis basé sur les résultats de Bassani et al. [10] ainsi que sur ceux d'Aklumdov, Gasanova et Nizametdinova qui ne sont pas contradictoires. Pour pouvoir dire que la structure de bande rend bien compte du spectre de réflectivité, il faudrait bien sûr calculer à partir du modèle $\varepsilon_{1}$ et $\varepsilon_{2}$ à toute fréquence. On peut malgré tout se satisfaire de la correspondance entre les pics de réflectivité et les singularités de la densité d'états conjuguée. De plus, ce modèle ne prétend pas rendre compte des transitions vers les hautes bandes de conduction (plus de $8 \mathrm{eV}$ au-dessus de la bande de valence). 